\title{
Endosomal recruitment of the WASH complex: active sequences and mutations impairing interaction with the retromer.
}

Running title: retromer-WASH complex interaction

Emmanuèle Helfer ${ }^{1, \pi}$, Michael E. Harbour ${ }^{2, \text {, }}$, Véronique Henriot ${ }^{1}$, Goran Lakisic $^{1}$, Carla Sousa-Blin ${ }^{1}$, Larisa Volceanov ${ }^{1}$, Matthew N.J. Seaman ${ }^{2, *}$, Alexis Gautreau, ${ }^{1, *}$

${ }^{1}$ Centre de Recherche de Gif, Laboratoire d'Enzymologie et Biochimie Structurales, CNRS UPR3082

Avenue de la Terrasse 91198 Gif-sur-Yvette Cedex, France.

${ }^{2}$ Department of Clinical Biochemistry, Cambridge Institute for Medical Research, University of Cambridge, Addenbrooke's Hospital, Cambridge CB2 OXY, U.K.

II Equal contribution

* Authors for correspondence:

mnjs100@cam.ac.uk or alexis.gautreau@lebs.cnrs-gif.fr

\section{ABSTRACT}

Background information. The WASH complex is the major Arp2/3 activator at the surface of endosomes. The branched actin network, that the WASH complex induces, contributes to cargo sorting and scission of transport intermediates destined for most endosomal routes. A major challenge is to understand how the WASH molecular machine is recruited at the surface of endosomes. The retromer endosomal machinery has been proposed by us and others to play a role in this process.

Results. Here we used an unbiased approach to identify the endosomal receptor of the WASH complex. We have delineated a short fragment of the FAM21 subunit that is able to displace (C) 2013 Sogiété Francaise des Microscopies and Société de Biologie Cellulaire de France

This article has been accepted for publication and undergone full peer review but has not been through the copyediting, typesetting, pagination and proofreading process, which may lead to differences between this version and the Version of Record. Please cite this article as DOI:10.1111/boc. 201200038 
the endogenous WASH complex from endosomes. Using a proteomic approach we have identified the retromer Cargo Selective Complex (CSC) as a partner of the active FAM21 sequence displacing the endogenous WASH complex. A point mutation in FAM21 that abolishes CSC interaction also impairs WASH complex displacement activity. The CSC is composed of three subunits, VPS35, VPS29 and VPS26. FAM21 directly binds the VPS35 subunit of the retromer CSC. Additionally, we show that a point mutant of VPS35 that blocks binding to VPS29 also prevents association with FAM21 and the WASH complex revealing a novel role for the VPS35-VPS29 interaction in regulating retromer association with the WASH complex.

Conclusions. This novel approach of endogenous WASH displacement confirms previous suggestions that the retromer is the receptor of the WASH complex at the surface of endosomes and identify key residues that mediate this interaction. The interaction between these two endosomal machineries, the WASH complex and the retromer, is likely to play a critical role in forming platforms at the surface of endosomes for efficient sorting of cargoes.

Keywords: Retromer, Arp2/3 complex, VPS29, VPS35, FAM21

\section{INTRODUCTION}

Actin polymerization is critical for many fundamental functions in the eukaryotic cell including cell migration and intracellular trafficking (Firat-Karalar and Welch, 2011). The Arp $2 / 3$ complex is the only known machinery that generates branched actin networks, functioning to nucleate a new actin filament from the side of prexisting actin filaments (Pollard, 2007). The activation of the Arp2/3 complex requires a class of proteins called Nucleation Promoting Factors (NPFs) that are characterized by a C-terminal VCA domain. Four families of NPFs are present in mammalian genomes: N-WASP, WAVE, WHAMM and WASH (Rottner et al., 2010). The WASH NPF is present at the surface of endosomes, where it plays a major role in the recruitement of the Arp2/3 complex (Derivery et al., 2009b; Gomez and Billadeau, 2009; Duleh and Welch, 2010).

The function of WASH and of the actin networks it promotes is important for three major endosomal trafficking routes. WASH depletion affects cargoes that are recycled, such as the transferrin receptor (Derivery et al., 2009b), $\alpha 5 \beta 1$ integrin (Zech et al., 2011), and $\beta 2$ adrenergic receptor (Puthenveedu et al., 2010). It affects the retrograde pathway of the cation- 
independent mannose 6 phosphate receptor (Gomez and Billadeau, 2009; Harbour et al., 2010). It also affects lysosomal targeting of EGF (Duleh and Welch, 2010) and, in Dictyostelium, the retrieval of v-ATPase (Carnell et al., 2011) from the degradative pathway. WASH is involved in all these processes, presumably because it is critical for a general function shared by all these pathways. When WASH is depleted, increased endosomal tubules are observed possibly as a result of defective scission of transport intermediates emanating from endosomes (Derivery et al., 2009b; Gomez and Billadeau, 2009). Consistent with this hypothesis, WASH has been found associated with dynamin (Derivery et al., 2009b). For the $\beta 2$-adrenergic receptor, the branched actin network induced by WASH has also been found to be instrumental at an earlier step during cargo sorting in endosomal tubules (Puthenveedu et al., 2010).

WASH is part of a stable multiprotein complex that has been purified by tandem affinity purification (Derivery et al., 2009b). This complex contains 5 core subunits: WASH, FAM21, Ccdc53, Strumpellin and SWIP (also known as KIAA1033) and associates with the pre-existing complex heterodimer of Capping Protein that blocks the elongation of actin filaments (Derivery et al., 2009b; Jia et al., 2010). FAM21 is composed of a small ( 200 amino acids) N-terminal domain, that is embedded within the WASH complex and a long ( 1100 amino acids) unstructured C-terminal tail (Gomez and Billadeau, 2009; Derivery and Gautreau, 2010). The long unstructured tail of FAM21 binds to the surface of endosomes (Gomez and Billadeau, 2009; Harbour et al., 2012).

WASH has a complex interplay with another endosomal sorting machinery, the retromer complex. Retromer is a conserved endosomal coat that mediates retrograde transport, from endosomes to the Golgi. Notable retromer cargoes include Vps10p in yeast and the cation independent mannose 6 phosphate receptor in mammalian cells (Seaman et al., 1997; Seaman et al., 1998; Seaman, 2004). The mammalian retromer is composed of two complexes; the sorting nexin (SNX) subcomplex that comprises a dimer of SNX-BAR proteins and the Cargo Selective Complex (CSC), that is composed of VPS35, VPS29 and VPS26 proteins (Bonifacino and Hurley, 2008). Endosomal tubules formed upon WASH complex depletion are stained by both complexes of the retromer (Gomez and Billadeau, 2009; Harbour et al., 2010). Furthermore, the depletion of the CSC, but not the SNX-BAR complex, prevents association of the WASH complex with endosomes (Harbour et al., 2010). We have recently shown that the FAM21 tail that associates with endosomes, binds the CSC of retromer through its VPS35 subunit (Harbour et al., 2012). All together, these experiments suggested that the CSC could be an endosomal receptor for the WASH complex. However, 
this idea seems contrary to the observation that the WASH complex has a general role in all major endosomal routes, whereas the retromer complex is strongly associated with the retrograde pathway.

In order to identify the general receptor of the WASH complex at the surface of endosomes, we have built upon the previous observation that the long FAM21 C-terminal tail associates with endosomes. We have defined a small FAM21 fragment that efficiently displaces endogenous WASH from endosomes and identified the proteins recognized by this fragment using proteomics. This new approach allowed us to confirm the retromer CSC as the receptor of the WASH complex. Furthermore, using a novel point mutation of VPS35, we show that this interaction between the WASH complex and its receptor requires the VPS35VPS29 interaction, but not the VPS35-VPS26 interaction. 


\section{RESULTS}

\section{Delineation of a short region of the FAM21 tail that can displace endogenous WASH from endosomes when overexpressed}

We have previously shown that the endosomal recruitment of the WASH complex at the surface of endosomes requires the retromer (Harbour et al., 2010; Harbour et al., 2012). However, this requirement does not prove that the retromer is the sole receptor of the WASH complex at the surface of endosomes. Therefore we decided to build on the previous observation that FAM21 tail associates with endosomes (Gomez and Billadeau, 2009). We used transient transfection of FAM21 fragments in 3T3 cells to examine whether these fragments can displace endogenous WASH. Such an activity would suggest that the active FAM21 fragment is saturating the endosomal receptor of the WASH complex.

In our assay, proteins were fused at their N-terminus with PC-mCherry fluorescent protein. 3T3 cells were transiently transfected and after two days of expression, fixed and processed for WASH immunofluorescence (Fig. 1A). In control cells transfected with PCmCherry, we observed a punctate localization of WASH by standard epifluorescence. When PC-mCherry-FAM21 was overexpressed, WASH became more diffuse. To confirm that FAM21 overexpression displaces endogenous WASH, we used confocal microscopy. Transfected cells were loaded with fluorescent Transferrin (Tf) to label recycling and early endosomes, on which WASH is enriched. In control cells, we observed the WASH localisation on the side of Tf-positive endosomes that we previously reported (Derivery et al., 2009b; Derivery et al., 2012). In contrast, when FAM21 was overexpressed, WASH staining was indeed more diffuse. But on single confocal planes, WASH punctae were still clearly visible. These small aggregates however were not associated with endosomes (Fig. 1B). This experiment indicates that indeed FAM21 overexpression displaces endogenous WASH from the endosomes.

We then used this assay to delineate the active fragment of FAM21. The short Nterminal domain of FAM21 (amino-acids 1-228, Fig. 2B), which is connected to the WASH complex, does not display such an activity, whereas the C-terminal FAM21 tail (amino-acids 229-1341) has an activity similar to the full length molecule. This result is in line with the observation that the FAM21 tail associates with endosomes (Gomez and Billadeau, 2009). To 
quantify the potency of each fragment, we counted the number of cells displaying WASH displacement. In all assays, the majority of the cells displayed a non-ambiguous phenotype of displacement or non-displacement corresponding to a cytosolic or punctate WASH staining by epifluorescence. However, in some cells, WASH displacement was ambiguous and thus we created a third category of ambiguous displacement in order to maintain strict inclusion criteria in the two former categories. The C-terminal tail of FAM21 is predicted to be unstructured (Derivery and Gautreau, 2010). So we divided the FAM21 tail in 3 roughly equal parts, paying attention not to interrupt conserved regions of homology revealed in alignments of multiple FAM21 sequences. These 3 parts were referred to as U1, U2 and U3, for Unstructured (Fig. 2A). Even though U1 and U2 were slightly more active than the negative control, U3 was by far the most effective fragment displaying endogenous WASH displacement (Fig. 2B). U3 was then further subdivided in 3 other roughly equal parts, U3A, U3B and U3C (Fig. 2A). U3A retained only a low displacement activity. U3B was completely inactive. U3C, the fragment composed of the last $144 \mathrm{C}$-terminal amino-acids, was the most effective at displacing endogenous WASH, although it was marginally less active compared to $\mathrm{U} 3$.

The detection of all PC-mCherry fusion proteins by anti-PC western blot indicated that all fusion proteins were of the expected size (Fig. 2C). Moreover, it revealed differences in levels of expression, which correlated with the difficulty we had at finding cells transfected with some contructs. Strikingly, all constructs that efficiently displaced WASH from endosomes (FL, Cter, U3, and U3C) also displayed relatively low expression compared to the others. Moreover, the most potent WASH-displacing constructs (FL and C-ter) were expressed much less than the constructs that displaced WASH comparatively less efficiently (U3 and U3C). Two further transfection experiments had to be used with the active constructs to collect sufficient data for comparison with the other constructs. This strong correlation suggested that the mislocalization of the endosomal WASH complex is toxic to $3 \mathrm{~T} 3$ cells. The U3C fragment was a good compromise of an active fragment with relatively good expression.

\section{Identification of the putative endosomal receptors of the WASH complex}

We next aimed to identify partners of the U3C fragment using proteomics. To this end, we produced and purified U3C in E.coli. We then coupled this FAM21 fragment to resin to create an affinity column. A large amount of HeLa cell extract (derived from $3 \mathrm{~L}$ of culture) was passed over the U3C affinity column. The column was then washed and eluted with high 
salt using an FPLC system. Eluted proteins were identified by LC-MS/MS (Fig. 3A and Supplementary Fig. S1). The most intense band was the mitochondrial dienoyl coA reductase. Since this protein and the WASH complex do not localize to the same compartment, this interaction is likely an artifact due to cell lysis. There were also common contaminants of affinity chromatography like cyclophilin, actin and myosin. The proteins that attracted our attention were subunits of the retromer, VPS35 and VPS26.

We have previously reported the co-immunoprecipitation of the WASH complex with the CSC subcomplex (Harbour et al., 2010), whereas Gomez and Billadeau had reported the co-immunoprecipitation of the WASH complex with the SNX subcomplex (Gomez and Billadeau, 2009). We established 3T3 stable cell lines expressing PC-GFP fusions of SNX1 and SNX2 from the same locus using FRT homologous recombination. A similar 3T3 stable cell line expressing PC-GFP-VPS35 was previously reported (Derivery et al., 2012). The SNX1 fusion protein was little expressed (data not shown), whereas SNX2 and VPS35 fusion proteins were well and similarly expressed (Fig. 3B). We thus used these two cell lines to validate the interaction of $\mathrm{U} 3 \mathrm{C}$ with the retromer. Using a GST-U3C pull-down, we found an interaction of FAM21 with the CSC complex, but not the SNX complex (Fig. 3C), consistent with our previous results.

\section{A point mutation in $\mathrm{U} 3 \mathrm{C}$ that impairs the interaction with the CSC complex also impairs its ability to displace endogenous WASH}

This independent approach of WASH displacement thus pointed at the retromer CSC as the potential receptor of the WASH complex. To confirm this role in our system, we sought to identify point mutations that would abolish CSC binding. FAM21 homologues from diverse species were aligned, the alignment of the U3C fragment is displayed in Supplementary Fig. S2. Only a few amino-acids are conserved in all species. We designed six point mutations, which either substituted phenylalanines for alanines or reversed the charge of acidic residues by replacing aspartic acid with arginine.

We purified GST-fusions of all six mutated U3C and performed a pull-down assay in PC-GFP-VPS35 cell line extracts as above (Fig. 4A, the numbering is according to FL FAM21). Three mutations (F1270A, D1272R, and D1297R) had no effect. The next two mutations (F1299A and F1331A) markedly reduced the amount of retained VPS35. The most C-terminal mutation, D1333R, totally abolished binding to the CSC. We verified that this mutation does not change the secondary structure of the U3C fragment. Circular dichroism 
confirmed that U3C is indeed unstructured, in agreement with the predictions. The D1333R mutation does not significantly change this characteristic of U3C (Fig. 4B). This experiment suggests that the D1333R mutation directly affects the CSC binding site in U3C.

We then tested whether this mutation impairs the ability of U3C to displace endogenous WASH. D1333R U3C was much less able to displace WASH from endosomes than WT U3C, although residual activity was still present (Fig. 5A-C). Strikingly, The D1333R mutation induced a much better expression of U3C in 3T3 cells, consistent with the previously described correlation between WASH displacement and toxicity (Fig. 5D). We also inserted this mutation into FL FAM21 in order to test whether this single point mutation significantly impairs WASH displacement. However, in the context of the full length molecule, not a single $3 \mathrm{~T} 3$ transfected cell could be observed in the microscope and the fusion protein could not be detected by Western Blot. This point mutation exerts different effects on toxicity in different FAM21 constructs.

As the results above suggest that the CSC is the endosomal receptor of the WASH complex, we sought to directly observe WASH and CSC colocalization when active fragments of FAM21 were overexpressed. Transfected 3T3 cells were observed by confocal microscopy. In control cells, WASH and VPS35 punctae indeed quantitatively colocalize (Fig. 6). When FAM21 FL and U3C are overexpressed, WASH staining displayed numerous small punctae that did no longer associate with VPS35 punctae. The D1333R mutation that impaired the ability of $\mathrm{U} 3 \mathrm{C}$ to displace WASH from endosomes partially restored the association between WASH and VPS35. Altogether these results indicate that the CSC fulfills the expected properties of an endosomal receptor for the WASH complex.

\section{Additional binding sites for the CSC complex along the FAM21 tail}

The interaction between FAM21 and the retromer CSC was further studied by the analysis of their co-immunoprecipitation using the various FAM21 fragments that were used in the displacement assay. For this experiment, HeLa cells, which are more efficiently transfected than $3 \mathrm{~T} 3$ cells, were used. The exogenous mCherry fusion proteins were all detected by coomasssie blue staining of the immunoprecipitates (Fig. 7). Strumpellin, another subunit of the WASH complex, was detected by western blot only if FAM21 N-terminal domain was present (e.g. lanes 2 and 3) consistent with the previous suggestion that the Nterminal domain of FAM21 is critical for FAM21 incorporation into the WASH complex (Gomez and Billadeau, 2009). The entire FAM21 tail efficiently precipitated the retromer 
CSC (lane 4). The FAM21 tail fragments that were best able to displace endogenous WASH in $3 \mathrm{~T} 3$ cells, such as Cter, U3, and U3C in lanes 4, 7, and 10, also interacted strongly with the CSC. The fragments which had a residual activity, such as U1 and U2 in lanes 5 and 6, also interacted weakly with the CSC. U3B which had no displacement activity did not interact at all with the CSC (lane 9). Only U3A displayed a residual displacement activity and was not able to precipitate the CSC (lane 8). In other words, the correlation between CSC binding and WASH displacement is very good. With the only exception of U3A, all FAM21 constructs would bind an amount of CSC proportional to their WASH displacement ability. Conversely, when the impairing mutation D1333R was introduced in U3C, it did strongly decrease CSC association (lane 11), but it did not completely abrogate it, in agreement with its residual displacement activity (Figs. 5C and 6). When the D1333R mutation was introduced into full length FAM21, FAM21 was less expressed, as in 3T3 cells, and we had to transfect more HeLa cells to compare the mutant construct to the wild type. The CSC was still associated to D1333R FAM21 consistent with the presence of several additional CSC binding sites along the FAM21 tail (lane 12).

In conclusion, even though we mapped the strongest binding site in $\mathrm{U} 3 \mathrm{C}$, there are numerous additional CSC binding sites along the FAM21 tail. The association of FAM21 with the CSC correlates tightly with the localisation of WASH on the endosomes, further reinforcing the strength of evidence that the retromer CSC is the receptor of the WASH complex at the surface of endosomes.

\section{Binding of VPS35 to the WASH complex requires its association with VPS29 but not with VPS26}

In early studies of Vps35p function in endosome-to-Golgi retrieval a temperature conditional allele of vps35 in Saccharomyces cerevisiae was reported (Seaman et al., 1997), but the nature of the mutation(s) was not identified at the time. We sequenced this allele and found a single mutation, the His742Arg substitution in Vps35p. This histidine residue is perfectly conserved in orthologous VPS35 genes and we have used the crystal structure of the human VPS35-VPS29 complex (Hierro et al., 2007) to map the localisation of the corresponding His675. The His675 residue lies on an $\alpha$-helix in the concave surface formed by HEAT repeats of VPS35 (Fig. 8A). Strikingly, His675 is located at the interface with VPS29, making contacts with Ile91 of VPS29, a residue that was previously shown to be 
required for the VPS29-VPS35 interaction (Collins et al., 2005). These contacts include hydrophobic contacts and a hydrogen bond (Fig. 8B).

We thus transiently transfected HeLa cells with GFP fusions of VPS35 wildtype, H675R or L108P, a previously characterized mutation that prevents binding of VPS35 to VPS26 (Gokool et al., 2007). Through anti-GFP immunoprecipitations we assessed whether these mutated forms bind to the other subunits of the CSC, VPS26 and VPS29. As anticipated from the crystal structure of the complex, the H675R mutation impairs binding of VPS35 to VPS29, but does not affect binding to VPS26 (Fig. 9). Conversely, the L108P mutation prevents binding of VPS35 to VPS26, but does not affect binding to VPS29. This pair of VPS35 mutations therefore provides a means to selectively uncouple VPS35 from VPS29 or VPS26.

Our previous data has shown that FAM21 binds to the VPS35 subunit of the retromer CSC in a yeast two-hybrid assay (Harbour et al., 2012). In order to examine the importance of the whole CSC complex in the interaction with the WASH complex, we used this pair of VPS35 mutations. The GFP-VPS35 wildtype and L108P co-precipitated with the WASH complex subunits, FAM21, Strumpellin, WASH, whereas GFP-VPS35 H675R did not associate with the WASH complex. This experiment suggests that VPS29 binding to VPS35 is required for the retromer CSC to interact with the WASH complex.

To further test the idea, we introduced mutations into VPS29 that impair binding to VPS35. Residues Val90 and Ile91 of VPS29 were mutated into Asp or Ser respectively to impair binding of VPS29 to VPS35 (Collins et al., 2005). Mutation of Ile91, that makes extensive contacts with VPS35 completely abolished VPS35 binding (Fig. 10). Mutation of Val90, which does not make direct contact with VPS35 (Fig. 8B) induced a severe but incomplete defect. As expected, these two mutations similarly affected WASH complex binding (lanes 4 and 7 vs. lane 1). Conversely, another mutation of VPS29, L152E, which was previously established to inhibit binding to the RabGAP TBC1D5, but does not affect VPS35 binding (Harbour et al., 2010), had no effect on WASH complex interaction.

We sought to restore binding of mutated VPS29 by overexpressing VPS35. Indeed when VPS35 WT was overexpressed, VPS29 V90D bound to VPS35 and the GFP-VPS29 V90D mutant was then able to co-precipitate the WASH complex (lane 5 vs. lane 4). However, overexpression of VPS35 H675R did not restore binding to VPS29 V90D (lane 6). Overexpression of VPS35 WT did not however restore binding to VPS29 I91S as the Ile91 residue of VPS29 is critical for binding VPS35 through molecular interaction with His675 of VPS35 (Fig.8). All together, these data strongly suggest that the binding of the WASH 
complex to the VPS35 subunit of the CSC depends on the VPS35-VPS29 interaction, but not on the VPS35-VPS26 interaction.

\section{DISCUSSION}

Our results confirm that the retromer CSC is the receptor of the WASH complex at the surface of endosomes. We took the approach of delineating a small fragment of FAM21 tail that displaces the endogenous WASH complex from endosomes and identified the ligands of this fragment by proteomics. Among these ligands, the retromer CSC was identified. This unbiased approach reinforces the idea that the CSC is the receptor of WASH. Indeed, we had previously shown that the CSC co-immunoprecipitates with the WASH complex and most importantly that WASH is redistributed to the cytosol upon CSC knock-down (Harbour et al., 2010). The idea that the retromer is the receptor of the WASH complex is cemented by the observation that a single mutation in the U3C fragment that strongly impairs its interaction with CSC also impair its ability to displace endogenous WASH from endosomes.

Recently, the role of the retromer has been extended beyond its first identified role in the retrograde pathway. Retromer involvement in the endosome to cell surface recycling of the $\beta 2$ adrenergic receptor (Temkin et al., 2011), of the Wntless receptor (Harterink et al., 2011; Zhang et al., 2011) and of Crumbs (Pocha et al., 2011; Zhou et al., 2011) has been reported. These new data indicate that the retromer might have a more general function similar to the one ascribed to the WASH complex. In agreement with a role in cargo sorting, the WASH complex localizes to a restricted domain of endosomes (Derivery et al., 2009b; Gomez and Billadeau, 2009; Duleh and Welch, 2010). The size of the WASH domain is regulated by the branched actin network that WASH generates and we have shown recently that the CSC responds similarly, consistent with the idea that these two interacting machineries form a sorting platform at the surface of endosomes (Derivery et al., 2012).

While our manuscript was in preparation, a publication by Rosen and colleagues reported the identification of numerous repeated motifs, called LFa motifs, in the FAM21 tail that interact with VPS35 (Jia et al., 2012). Our data generally agree with these findings. For example, the point mutation D1333R of the U3C that impairs CSC binding falls within an LFa motif (Fig.S2). Another observation consistent with the numerous LFa motifs is that fragments of FAM21 tail outside of U3C still bind to the CSC. However, several of our observations cannot be accounted by this recent publication. The U3C contains the 5 last LFa 
motifs (from 17 to 21 ). The most efficient mutations in our analysis of the U3C binding to the CSC affect the $21^{\text {st }}$ LFa motif, F1331A and D1333R. In contrast, our mutations of conserved residues that affect the $20^{\text {th }}$ LFa motif, D1297R and 1299A, have a moderate effect. This is in contrast with the report of Jia et al., that showed that the $20^{\text {th }} \mathrm{LFa}$ is stronger than the $21^{\text {st }} \mathrm{LFa}$ motif. We observe other discrepancies when we analyse our delineation of the active fragment in the light of LFa motifs. For example, U3B contained two LFa repeats but had no effect on WASH localization and did not bind to the CSC. These discrepancies call for a refinement of the structural motifs that mediate the interaction of FAM21 with VPS35.

Our analysis of the retromer - WASH complex interaction supports a critical role of the VPS29-VPS35 interaction. We had initially reported the FAM21-VPS35 interaction in the yeast two hybrid assay (Harbour et al., 2010). This direct interaction was recently confirmed by a GST-FAM21 tail pull-down using recombinant VPS proteins (Jia et al., 2012). So there is no doubt that FAM21 binds directly to VPS35. However, our analyses of the native interactions using immunoprecipitations argue that VPS35 must be bound to VPS29 to interact with the WASH complex. This role of VPS29 was not observed in vitro by Jia et al. We established the requirement for VPS29 in the interaction with the WASH complex using a newly identified mutation of human VPS35, H675R, based upon a temperature sensitive allele of yeast vps35. This mutation abolishes the binding to both VPS29 and WASH complex. Similarly, the rescue of the VPS29 mutation, V90D, by the overexpression of WT VPS35, but not by H675R, restored both the binding to both VPS35 and WASH complex. This requirement of VPS29 is thus strongly supported by these several means to modulate the VPS29-VPS35 interaction.

We can only speculate as to why VPS29 binding would be required for the VPS35FAM21 interaction. The CSC is a stable tripartite complex that has been reconstituted (Hierro et al., 2007). VPS35 binds to VPS29 and VPS26 independently and the affinity of VPS35 for VPS29 is $\sim 60$ fold less than the affinity for VPS26 (Collins, 2008; Norwood et al., 2011), raising the intriguing possibility that VPS29 association and dissociation on and off the VPS35 platform regulates the interaction with the WASH complex. A 'rigidification' of VPS35 has been proposed upon VPS29 binding (Norwood et al., 2011). It is thus a possibility that VPS29 binding favors a conformation of VPS35 that supports FAM21 binding. VPS29 is itself a scaffold protein that weakly connects the CSC complex to the SNX-BAR complex of the retromer (Swarbrick et al., 2011). It is thus possible that the interaction with the WASH complex and, as a consequence, local actin polymerization occurs only with fully assembled retromer, when it is actively involved in cargo sorting. 
In conclusion, our present results not only confirm that the retromer is the receptor of the WASH complex at the surface of endosomes but also defines two point mutations, the D1333R mutation of FAM21 and the H675R substitution of VPS35, that clearly regulate this important VPS35-FAM21 interaction. Our results also illustrate the complexity of this molecular system, as there is more than one binding site for VPS35 on the FAM21 tail and that the H675R mutation of VPS35 affects FAM21 binding through VPS29 rather than by affecting directly the unknown binding site of VPS35 for the FAM21 tail. Further studies combining cell biology observations with in vitro reconstitutions and structural biology are required to draw a precise scenario of the molecular events yielding efficient and accurate sorting of specific cargoes. 


\section{MATERIALS AND METHODS}

\section{Plasmids and antibodies}

FAM21 ORF was amplified by PCR from previsouly described cDNAs (Gomez and Billadeau, 2009; Harbour et al., 2010). FAM21 constructs were cloned into either the pcDNAm FRT PC mCherry vector between the FseI and AscI restriction sites or into the pEGFP C1 vector between the BamHI and SalI sites. All PCR amplified fragments were sequenced to ensure that no unwanted mutations were introduced. pcDNAm FRT PC mCherry drives the expression of fusion proteins C-terminal to a Protein C (PC) tag and a mCherry tag under the control of a EF1 $\alpha /$ HTLV chimera promoter. For $3 \mathrm{~T} 3$ transfections, plasmids were purified using the Nucleobond PC 20 kit (Macherey-Nagel).

Human SNX1 and SNX2 were amplified from templates provided by Ludger Johannes (Institut Curie, Paris) and cloned into the pcDNA5 FRT PC GFP vector. pcDNA5 FRT PC GFP drives the expression of fusion proteins C-terminal to a Protein C (PC) tag and eGFP.

pGEX-CS-U3C was constructed by shuttling U3C from the mCherry vector into pGEX-CS which encodes GST fusion with a TEV cleavage site. The GFP-VPS35 wildtype and L108P constructs have been described previously (Gokool et al., 2007). Wildtype VPS35 and the VPS35 H675R mutant were subcloned into pmCherry C1. Site directed mutagenesis was performed using Quikchange kit (Stratagene).

The PC mAb (HPC-4) was purchased from Roche. Anti-tubulin mAb (clone E7) was developed by M. Klymkowsky and obtained from Developmental Studies Hybridoma Bank. Antibodies against VPS26, VPS35, Strumpellin, Fam21 used for western blotting have been described previously in (Seaman, 2004; Harbour et al., 2010; Harbour et al., 2012). The VPS35 mAb (B-5) used for immunofluorescence in Fig. 8 was purchased from Santa Cruz Biotechnology. The anti-GFP used for immunoprecipitations and western blotting is described in (Seaman et al., 2009). Polyclonal anti-dsRed used to detect the mCherry constructs was purchased from Clontech.

\section{Cell culture, transfections, and stable cell lines}

3 T3 cells were grown in DMEM medium supplemented with $10 \%$ donor calf serum (DCS) without antibiotics and transiently transfected using either Lipofectamine 2000 (Invitrogen), 
GeneCellin (BioCellChallenge) or the Neon electroporator (Life Technologies). Stable Flp-In $3 \mathrm{~T} 3$ transfectants obtained by homologous recombination at the FRT site were obtained as previously described using Hygromycin selection (Derivery et al., 2009b). The 3T3 cell line expressing PC-GFP-VPS35 was previously described (Derivery et al., 2012).

HeLaM cells stably expressing VPS29-GFP wildtype and mutants have been described previously (Collins et al., 2005 ; Harbour et al., 2010). Transient transfection of HeLaM cells was performed using Polyethylenimine (PEI - Polysciences Inc) as the carrier following a protocol similar to Lipofectamine mediated transfections. Briefly: HeLaM cells at $\sim 50 \%$ confluency in $140 \mathrm{~mm}$ tissue culture dishes were transfected with $24 \mu \mathrm{g}$ of plasmid DNA complexed with $60 \mu \mathrm{l}$ of $10 \mathrm{mM}$ PEI in $3 \mathrm{ml}$ of Optimem (Invitrogen). After 20 mins incubation of the PEI and DNA, the mix was added dropwise to $14 \mathrm{ml}$ of prewarmed media (DMEM, 10\% fetal calf serum, antibiotics and glutamine) covering the cells in the tissue culture dish. The Optimem/media mix was gently swirled in the dish to mix and then the dish was returned to the tissue culture incubator. The cells were used for native IPs 48 hours post transfection.

\section{Native Immunoprecipitations}

The anti-mCherry and anti-GFP native immunoprecipitations were performed essentially as described in (Harbour et al., 2012). Briefly: the media covering cells in a $140 \mathrm{~mm}$ tissue culture dish was removed and the cells were washed with $10 \mathrm{ml}$ PBS. After removal of the PBS, the cells were placed on ice at an angle to drain for 5 mins. Any remaining PBS was removed and then cells were then scraped up in $1 \mathrm{ml}$ of chilled lysis buffer (20 mM Hepes$\mathrm{KOH}, \mathrm{pH} 7.2$, $50 \mathrm{mM}$ potassium acetate, $2 \mathrm{mM}$ EDTA, $200 \mathrm{mM}$ sorbitol, 0.1\% Triton X100). The lysate was cleared by centrifugation at $10,000 \mathrm{~g}$ for $5 \mathrm{mins}$ at $4^{\circ} \mathrm{C}$. The lysate was then incubated with $50 \mu \mathrm{l}$ of protein A-sepharose (25\% slurry) to remove most proteins that bind non-specifically. After 30 mins incubation at $4{ }^{\circ} \mathrm{C}$ on a rotating wheel, the lysate was cleared again by centrifugation at $10,000 \mathrm{~g}$ for $5 \mathrm{mins}$ at $4^{\circ} \mathrm{C}$. The lysate was then transferred to a fresh $1.5 \mathrm{ml}$ microfuge tube and incubated with anti-GFP (5 $\mu \mathrm{g}$ per $1 \mathrm{ml}$ of lysate) for $90 \mathrm{mins}$ at $4^{\circ} \mathrm{C}$ on a rotating wheel after which $50 \mu 1$ of protein A-sepharose was added. For mCherry precipitations, RFP-Trap beads (Chromotek) were used. After a further 60 min incubation on a rotating wheel at $4^{\circ} \mathrm{C}$, the sepharose was collected by centrifugation $(1 \min 10,000 \mathrm{~g})$, the supernatant removed by aspiration and then washed by addition of $1 \mathrm{ml}$ of lysis buffer. The sepharose was washed three more times and then desiccated in a SpeedVac. The dried 
sepharose was then resuspended into $50 \mu \mathrm{l}$ of SDS-PAGE sample buffer, heated to $90^{\circ} \mathrm{C}$ for 5 mins and then loaded onto either a $10 \%$ or $8 \%$ polyacrylamide gel for electrophoresis.

\section{SDS-PAGE and Western Blots}

For Figs. 2 and 5, total cell lysates were prepared by resuspending $10^{6}$ cells directly in SDSPAGE loading buffer. Viscosity of samples after boiling was reduced using Benzonase (Sigma). SDS-PAGE was performed using NuPAGE 4-12\% Bis-Tris gels (Invitrogen) transferred onto nitrocellulose membranes according to the manufacturer's instructions. Western blots were revealed using HRP coupled antibodies, Supersignal@ kit (Pierce / Thermo Scientific) and a Fuji LAS-3000 (Fujifilm).

For Figs. 7, 9, and 10, western blots were developed using ${ }^{125}$ I-protein A (purchased from Perkin Elmer) to avoid strong reaction with the denatured IgG of the IP. For Figs. 9-10, gels were silver stained using the BioRad silver stain kit according to manufacturer's instructions.

\section{Immunofluorescence}

$3 \mathrm{~T} 3$ cells were plated for $2 \mathrm{~h}$ at $37^{\circ} \mathrm{C}$ on glass coverslips coated with $5 \mu \mathrm{g} / \mathrm{ml}$ fibronectin (Sigma), then loaded with Alexa647-Transferrin (Invitrogen) until equilibrium when indicated, fixed in PBS-3\% paraformaldehyde, permeabilized with PBS-0.05\% saponin, then processed for immunofluorescence using our affinity purified polyclonal WASH antibodies (Derivery et al., 2009a). Samples were mounted in Prolong® Gold antifade reagent (Invitrogen).

For the quantitative analysis of WASH displacement by FAM21 constructs, fixed cells were imaged by epifluorescence microscopy on an Axio-observer Z1 microscope (Zeiss) equipped with an Orca- $\mathrm{R}^{2}$ camera (Hamamatsu) and operated by Axiovision software (Zeiss). Images were acquired with 63 x NA 1.4 oil immersion or 20x NA 0.8 air objectives.

Confocal optical sections were acquired on a Zeiss LSM510 confocal microscope (Zeiss), equipped with a 63x NA 1.4 oil immersion objective and run by ZEN 2008 software (Zeiss), or on a SP8X confocal microscope (Leica), equipped with a 63x NA 1.4 oil immersion objective and run by LAS AF software (Leica).

\section{Quantification of WASH association with Vps35}

WASH and VPS35 confocal images were segmented with the JaCoP plugin (Fabrice Cordelières, Institut Curie, Orsay) of ImageJ, after median filtering and background subtraction. For each WASH object, the distance to all VPS35 objects was calculated using 
their centers of mass and the minimal distance was isolated using Excel. WASH was scored as colocalized with VPS35 if this distance was less than the optical resolution of $290 \mathrm{~nm}$.

\section{Proteomics}

Partners of U3C were identified by affinity chromatography. U3C was expressed in BL21* (Invitrogen) in fusion with GST (in TB medium with $0.5 \mathrm{mM} \mathrm{IPTG}$ for $2 \mathrm{~h}$ at $37^{\circ} \mathrm{C}$ ) and was purified using glutathione-sepharose beads with a standard protocol. U3C was cleaved off GST using TEV protease and coupled to a HiTrap $1 \mathrm{~mL}$ (NHS activated HP; GE Healthcare) according to manufacturer's instructions. A frozen Hela S3 cell pellet of $15 \mathrm{~mL}$ was lysed in a total volume of $75 \mathrm{~mL}$ of lysis buffer (50 mM Tris $\mathrm{pH}$ 7.5, $150 \mathrm{mM} \mathrm{NaCl}, 1 \mathrm{mM}$ EDTA, 1 mM DTT, $0.5 \%$ Triton X-100, 5\% glycerol supplemented with protease inhibitors). This lysate was clarified by centrifugation and ultracentrifugation. $40 \mathrm{~mL}$ of extract was passed on the U3C affinity column using an AKTA purifier (GE Healthcare). The U3C affinity column was then washed extensively with PBS and eluted with high salt buffer (PBS adjusted to 1.5 $\mathrm{mM} \mathrm{NaCl}$ ). Elution fractions were run on a NuPAGE 4-12\% Bis-Tris gel (Invitrogen). The proteins bands were excised, digested with trypsin, and analyzed by nanoLC-MS/MS (Q/TOF Premier, Waters; nanoRSLC, Dionex) at the Mass Spectrometry facility of Imagif (Gif-surYvette).

\section{Circular dichroism}

U3C fragments cleaved off GST were analyzed in TEV cleavage buffer $(25 \mathrm{mM}$ Tris- $\mathrm{HCl} \mathrm{pH}$ 8, $50 \mathrm{mM} \mathrm{NaCl}, 1 \mathrm{mM}$ beta-mercaptoethanol, $1.25 \mathrm{mM} \mathrm{MgCl}_{2}$ ). Far-UV CD spectra were recorded at $20^{\circ} \mathrm{C}$ using a JASCO J-810 dichrograph equipped with a thermostated cell holder using a $0.01-\mathrm{cm}$ path length quartz cuvette. Each spectrum was the average of 10 acquisitions recorded in the 280-185 nm range with $0.5-\mathrm{nm}$ steps, a bandwidth of $1 \mathrm{~nm}$, and at a speed of $100 \mathrm{~nm} \min ^{-1}$. All spectra were buffer corrected and normalized to the mean residue weight ellipticity $(\theta \mathrm{MRW})\left[\mathrm{deg} \mathrm{cm}^{2} / \mathrm{dmole}\right]$ using the equation $\theta(\lambda) \mathrm{MRW}=\theta(\lambda) \mathrm{mdeg} / 10 \cdot \mathrm{c} \cdot \mathrm{n} \cdot \mathrm{d}$ where $\theta(\lambda)$ mdeg is the recorded spectra in millidegrees, $\mathrm{n}$ is the number of amino acid residues, $\mathrm{c}$ is the sample concentration in moles per liter and $d$ is the path length of the cuvette in centimeters. 


\section{Author contribution}

MNJS AG designed the research. EH MEH CS VH GL LV MNJS performed experiments. EH MNJS AG wrote the paper.

\section{Funding}

This work was supported by grants from the Agence Nationale de la Recherche ([ANR-09JCJC-0040] and [ANR-11 BSV2 014 01]) and from the Medical Research Council (G0701444).

\section{Acknowledgements}

We thank Julie Ménétrey (LEBS, Gif), Luc Bousset (LEBS, Gif) and Fabrice Cordelières (Institut Curie, Orsay) for their help with structural analysis, circular dichroism, and colocalization analysis. We thank the cloning and biophysical facilities of the LEBS, David Cornu and Manuela Argentini from the SiCAPS Mass Spectrometry facility (IMAGIF), Marie-Noëlle Soler and Sébastien Bellow from the Imaging Facility (IMAGIF). We thank Daniel Billadeau (Mayo Clinic, Rochester) and Ludger Johannes (Institut Curie, Paris) for kindly providing us with reagents. EH belongs to the CellTiss Consortium. 


\section{REFERENCES}

Bonifacino, J.S., and Hurley, J.H. (2008). Retromer. Curr Opin Cell Biol 20, 427-436

Carnell, M., Zech, T., Calaminus, S.D., Ura, S., Hagedorn, M., Johnston, S.A., May, R.C., Soldati, T., Machesky, L.M., and Insall, R.H. (2011). Actin polymerization driven by WASH causes VATPase retrieval and vesicle neutralization before exocytosis. J Cell Biol 193, 831-839

Collins, B.M. (2008). The structure and function of the retromer protein complex. Traffic 9, 18111822

Collins, B.M., Skinner, C.F., Watson, P.J., Seaman, M.N., and Owen, D.J. (2005). Vps29 has a phosphoesterase fold that acts as a protein interaction scaffold for retromer assembly. Nat Struct Mol Biol 12, 594-602

Derivery, E., Lombard, B., Loew, D., and Gautreau, A. (2009a). The Wave complex is intrinsically inactive. Cell Motil Cytoskeleton 66, 777-790

Derivery, E., Sousa, C., Gautier, J.J., Lombard, B., Loew, D., and Gautreau, A. (2009b). The Arp2/3 activator WASH controls the fission of endosomes through a large multiprotein complex. Dev Cell 17, 712-723

Derivery, E., and Gautreau, A. (2010). Evolutionary conservation of the WASH complex, an actin polymerization machine involved in endosomal fission. Commun Integr Biol 3, 227-230

Derivery, E., Helfer, E., Henriot, V., and Gautreau, A. (2012). Actin Polymerization Controls the Organization of WASH Domains at the Surface of Endosomes. PLoS ONE 7, e39774

Duleh, S.N., and Welch, M.D. (2010). WASH and the Arp2/3 complex regulate endosome shape and trafficking. Cytoskeleton 67, 193-206

Firat-Karalar, E.N., and Welch, M.D. (2011). New mechanisms and functions of actin nucleation. Curr Opin Cell Biol 23, 4-13

Gokool, S., Tattersall, D., Reddy, J.V., and Seaman, M.N. (2007). Identification of a conserved motif required for $\mathrm{Vps} 35 \mathrm{p} / \mathrm{Vps} 26 \mathrm{p}$ interaction and assembly of the retromer complex. Biochem $\mathbf{J}$ 408, 287-295

Gomez, T.S., and Billadeau, D.D. (2009). A FAM21-containing WASH complex regulates retromerdependent sorting. Dev Cell 17, 699-711

Harbour, M.E., Breusegem, S.Y., Antrobus, R., Freeman, C., Reid, E., and Seaman, M.N. (2010). The cargo-selective retromer complex is a recruiting hub for protein complexes that regulate endosomal tubule dynamics. J Cell Sci 123, 3703-3717

Harbour, M.E., Breusegem, S.Y., and Seaman, M.N. (2012). Recruitment of the endosomal WASH complex is mediated by the extended 'tail' of Fam21 binding to the retromer protein Vps35. Biochem J 442, 209-220

Harterink, M., Port, F., Lorenowicz, M.J., McGough, I.J., Silhankova, M., Betist, M.C., van Weering, J.R., van Heesbeen, R.G., Middelkoop, T.C., Basler, K., Cullen, P.J., and Korswagen, H.C. (2011). A SNX3-dependent retromer pathway mediates retrograde transport of the Wnt sorting receptor Wntless and is required for Wnt secretion. Nat Cell Biol 13, 914-923

Hierro, A., Rojas, A.L., Rojas, R., Murthy, N., Effantin, G., Kajava, A.V., Steven, A.C., Bonifacino, J.S., and Hurley, J.H. (2007). Functional architecture of the retromer cargo-recognition complex. Nature 449, 1063-1067

Jia, D., Gomez, T.S., Billadeau, D.D., and Rosen, M.K. (2012). Multiple repeat elements within the FAM21 tail link the WASH actin regulatory complex to the retromer. Mol Biol Cell 23, 23522361 
Jia, D., Gomez, T.S., Metlagel, Z., Umetani, J., Otwinowski, Z., Rosen, M.K., and Billadeau, D.D. (2010). WASH and WAVE actin regulators of the Wiskott-Aldrich syndrome protein (WASP) family are controlled by analogous structurally related complexes. Proc Natl Acad Sci U S A 107, 10442-10447

Norwood, S.J., Shaw, D.J., Cowieson, N.P., Owen, D.J., Teasdale, R.D., and Collins, B.M. (2011). Assembly and solution structure of the core retromer protein complex. Traffic 12, 56-71

Pocha, S.M., Wassmer, T., Niehage, C., Hoflack, B., and Knust, E. (2011). Retromer controls epithelial cell polarity by trafficking the apical determinant Crumbs. Curr Biol 21, 1111-1117

Pollard, T.D. (2007). Regulation of actin filament assembly by Arp2/3 complex and formins. Annu Rev Biophys Biomol Struct 36, 451-477

Puthenveedu, M.A., Lauffer, B., Temkin, P., Vistein, R., Carlton, P., Thorn, K., Taunton, J., Weiner, O.D., Parton, R.G., and von Zastrow, M. (2010). Sequence-dependent sorting of recycling proteins by actin-stabilized endosomal microdomains. Cell 143, 761-773

Rottner, K., Hanisch, J., and Campellone, K.G. (2010). WASH, WHAMM and JMY: regulation of Arp2/3 complex and beyond. Trends Cell Biol 20, 650-661

Seaman, M.N. (2004). Cargo-selective endosomal sorting for retrieval to the Golgi requires retromer. J Cell Biol 165, 111-122

Seaman, M.N., Harbour, M.E., Tattersall, D., Read, E., and Bright, N. (2009). Membrane recruitment of the cargo-selective retromer subcomplex is catalysed by the small GTPase Rab7 and inhibited by the Rab-GAP TBC1D5. J Cell Sci 122, 2371-2382

Seaman, M.N., Marcusson, E.G., Cereghino, J.L., and Emr, S.D. (1997). Endosome to Golgi retrieval of the vacuolar protein sorting receptor, Vps10p, requires the function of the VPS29, VPS30, and VPS35 gene products. J Cell Biol 137, 79-92

Seaman, M.N., McCaffery, J.M., and Emr, S.D. (1998). A membrane coat complex essential for endosome-to-Golgi retrograde transport in yeast. J Cell Biol 142, 665-681

Swarbrick, J.D., Shaw, D.J., Chhabra, S., Ghai, R., Valkov, E., Norwood, S.J., Seaman, M.N., and Collins, B.M. (2011). VPS29 is not an active metallo-phosphatase but is a rigid scaffold required for retromer interaction with accessory proteins. PLoS ONE 6, e20420

Temkin, P., Lauffer, B., Jager, S., Cimermancic, P., Krogan, N.J., and von Zastrow, M. (2011). SNX27 mediates retromer tubule entry and endosome-to-plasma membrane trafficking of signalling receptors. Nat Cell Biol 13, 715-721

Zech, T., Calaminus, S.D., Caswell, P., Spence, H.J., Carnell, M., Insall, R.H., Norman, J., and Machesky, L.M. (2011). The Arp2/3 activator WASH regulates alpha5beta1-integrin-mediated invasive migration. J Cell Sci 124, 3753-3759

Zhang, P., Wu, Y., Belenkaya, T.Y., and Lin, X. (2011). SNX3 controls Wingless/Wnt secretion through regulating retromer-dependent recycling of Wntless. Cell research 21, 1677-1690

Zhou, B., Wu, Y., and Lin, X. (2011). Retromer regulates apical-basal polarity through recycling Crumbs. Dev Biol 360, 87-95 


\section{FIGURE LEGENDS}

\section{Figure 1. Full length FAM21 displaces endogenous WASH from endosomes.}

3T3 cells were transiently transfected with PC-mCherry or PC-mCherry fused to full length FAM21 (FL). After 2 days, cells were processed for immunofluorescence using WASH antibodies (A) Transfected cells were observed by epifluorescence microscopy. In control cells, endogenous WASH displays a perinuclear punctate pattern. In contrast, endogenous WASH displays a diffuse cytosolic staining in cells overexpressing PC-mCherry-FAM21. This experience is referred to the WASH displacement assay in the text (B) Transfected cells were loaded with fluorescent transferrin (Tf) prior to immunofluorescence processing, and observation by confocal microscopy. A single confocal section is displayed. In control cells, WASH punctae are associated with Tf-positive endosomes. When FAM21 is overexpressed, WASH is more diffuse and the agregates are not associated with endosomes.. Scale bar: 10 $\mu \mathrm{m}$ ( $1 \mu \mathrm{m}$ in inserts).

A

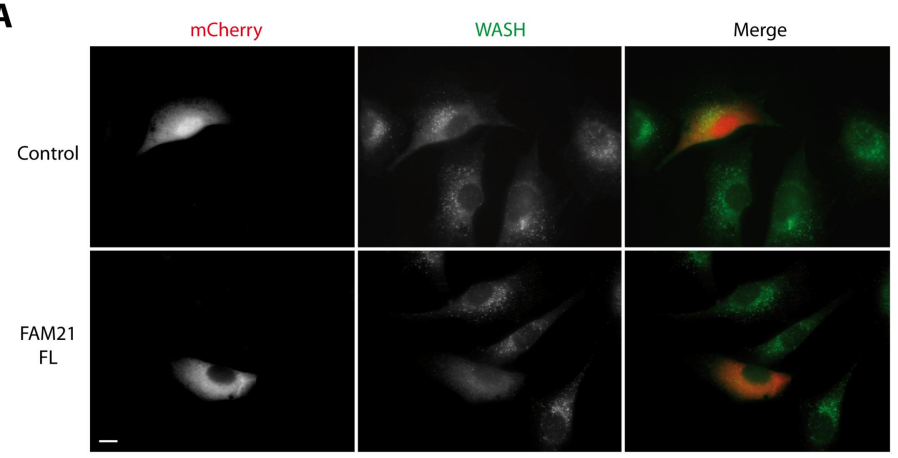

B

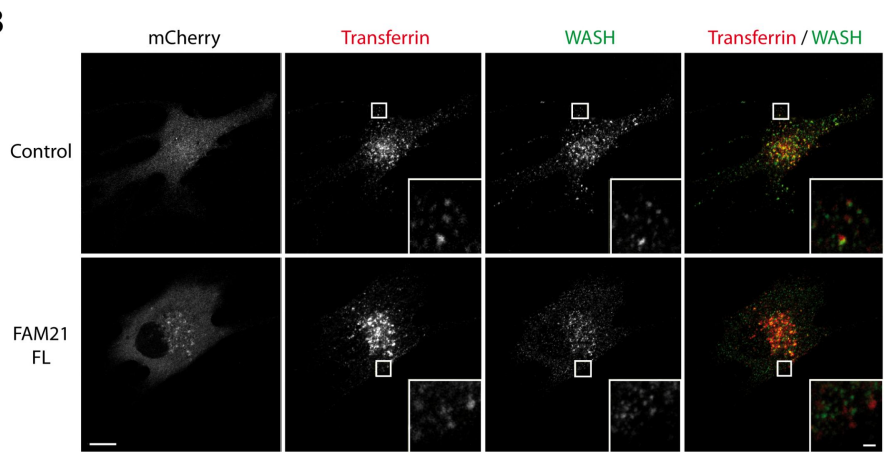


Figure 2. Delineation of a small FAM21 fragment, U3C, that efficiently displaces endogenous WASH from endosomes.

(A) Schematic representation of the different FAM21 constructs. All constructs are tagged with PC-mCherry fusion protein. (B) Percentages of cells displaying clear displacement of endosomal WASH (black), no displacement (white) or an ambiguous displacement (grey) (n: total number of transfected cells observed for each construct). The percentage values (mean +/- sem) were averaged over at least 2 independent experiments, in order to observe a minimum of 40 cells for each construct. The horizontal line represents the background level (from the control case) above which a construct is considered as able to displace WASH. (C) Detection of the different FAM21 constructs. A total cell lysate was prepared from transiently transfected 3T3 cells and immunoblotted with PC antibodies. All lanes are from a single blot, in which some intervening lanes were removed. Weak expression of some constructs correlates with the low number of cells expressing these constructs (see text for details).

A

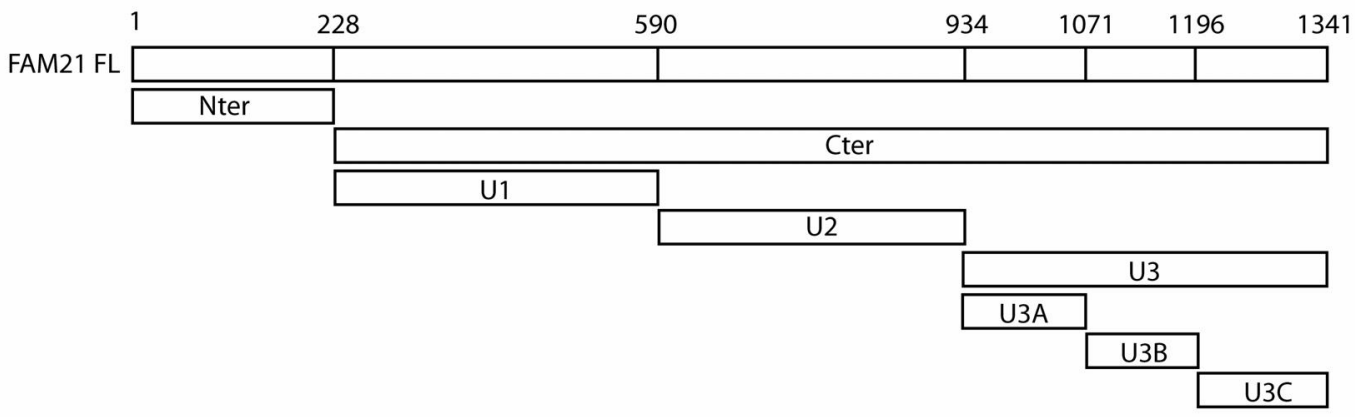

B

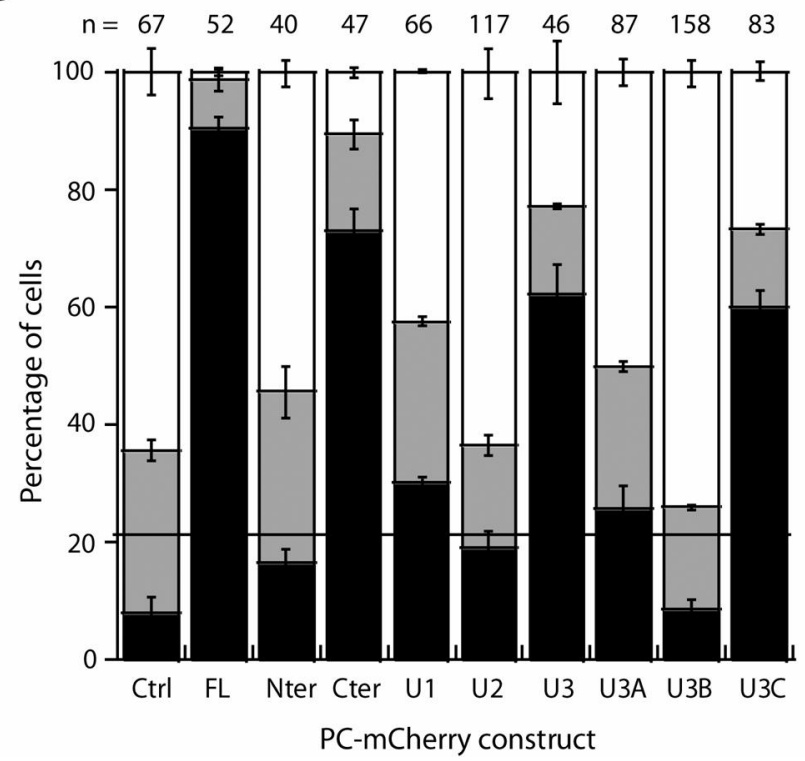

C

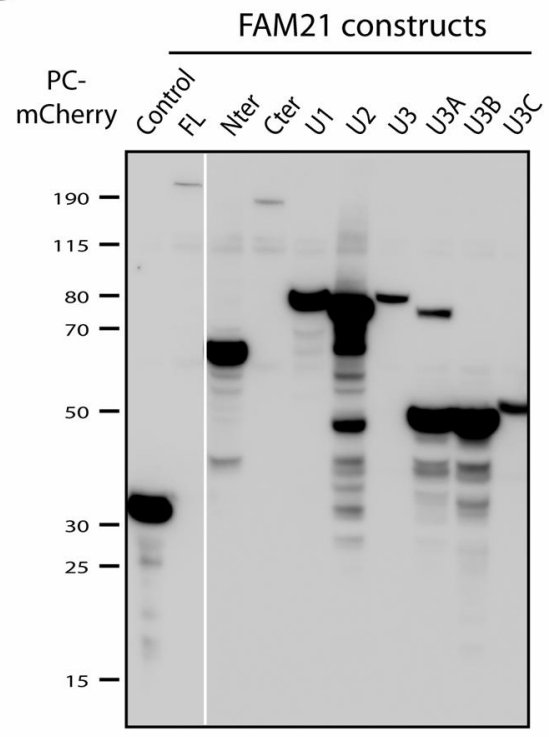


Figure 3. Proteomic analysis of $\mathrm{U} 3 \mathrm{C}$ binding partners reveals an interaction with the cargo selective complex of the retromer.

(A) A HeLa cell lysate was passed over an U3C affinity column, which was then eluted with high salt. Elution fractions were analysed by SDS-PAGE and stained with colloidal coomassie. Visible bands, numbered from 1 to 12 , were excised and analyzed by LC-MS/MS (Supplementary Fig. S1). The major proteins identified are indicated. (B) Stable 3T3 cell lines expressing PC-GFP tagged subunits of the retromer were established and characterized by immunoblotting total cell lysates using PC antibodies. (C) GST pull-down of U3C incubated with cell extracts from PC-GFP-VPS35 or PC-GFP-SNX2 cell lines. U3C binds to the Cargo Selective Complex of the retromer, but not to the SNX complex.

A

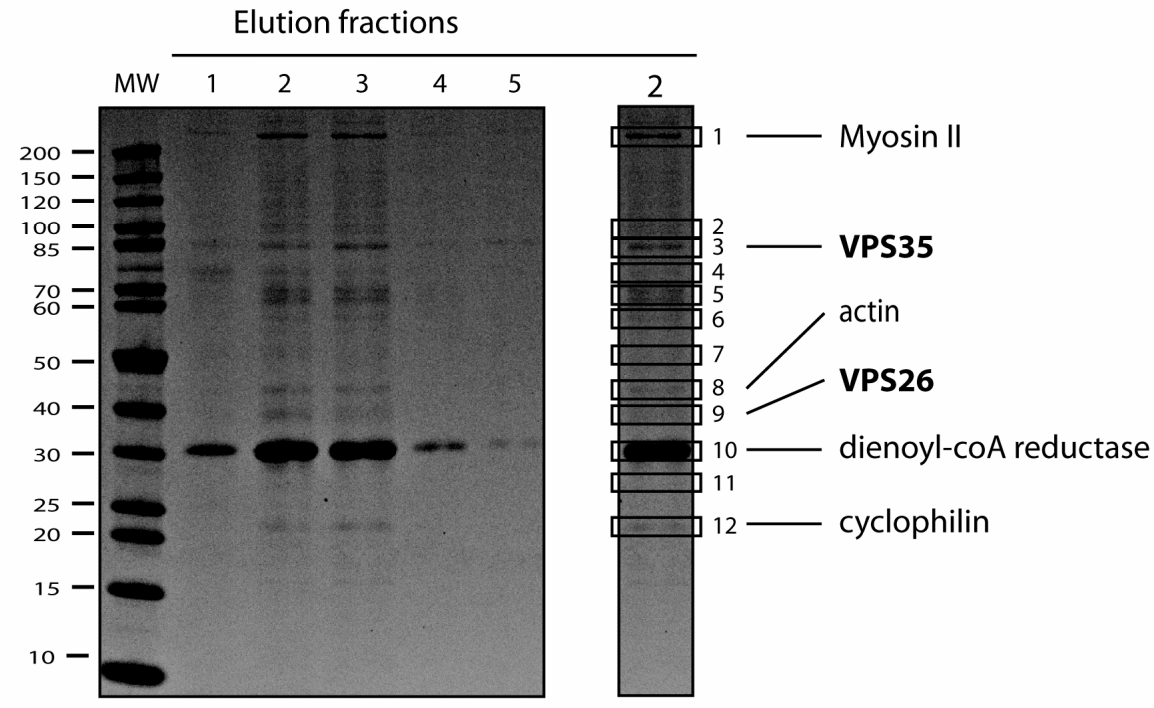

B
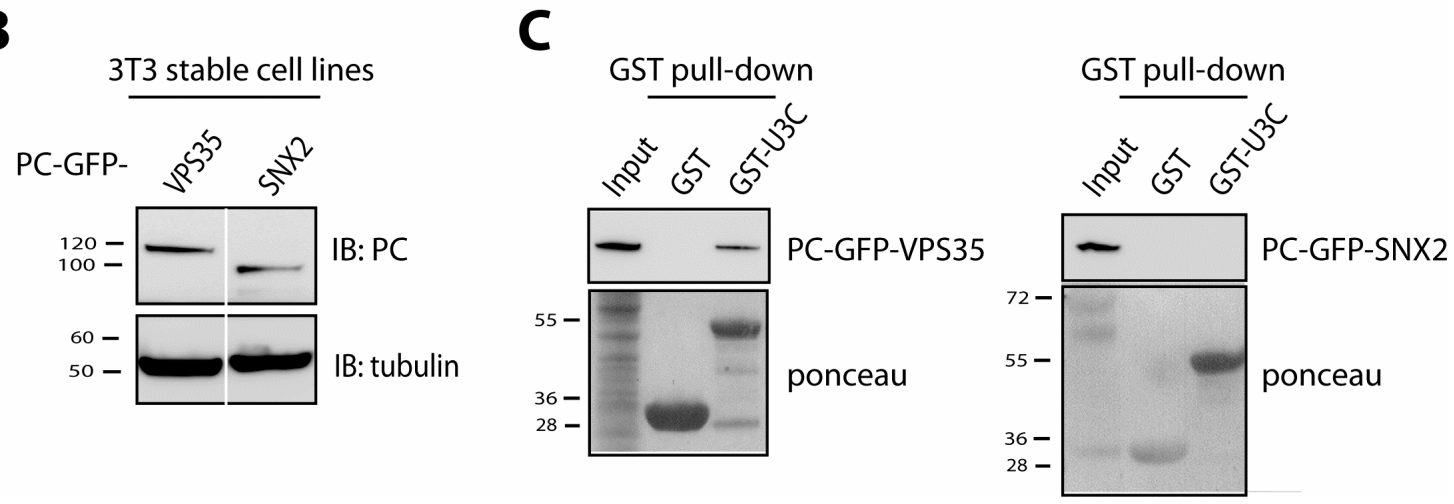


\section{Figure 4. Mutational analysis of U3C.}

(A) Mutations affecting conserved residues of FAM21 were introduced into U3C. These mutations either replaced phenylalanine residues by alanine or reversed the charge of aspartic acidic residues. Numbering is according to the full length FAM21. GST pull-down of the retromer using these mutants. The single point mutant D1333R abolishes binding to the Cargo Selective Complex. (B) Circular dichroism analysis of WT and mutant U3C. This spectrum is characteristic of unstructured regions. The spectrum is not affected by the D1333R mutation. This analysis confirms that the mutation affects a retromer binding motif and not the folding of a globular domain.

\section{A}

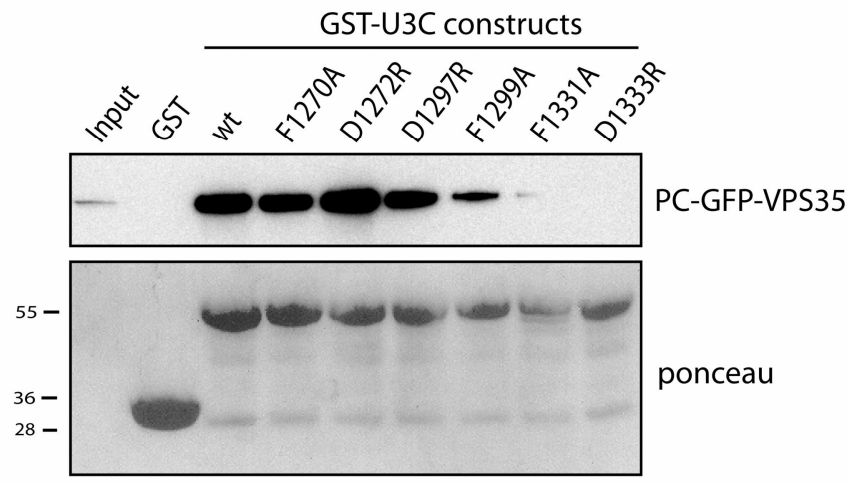

B

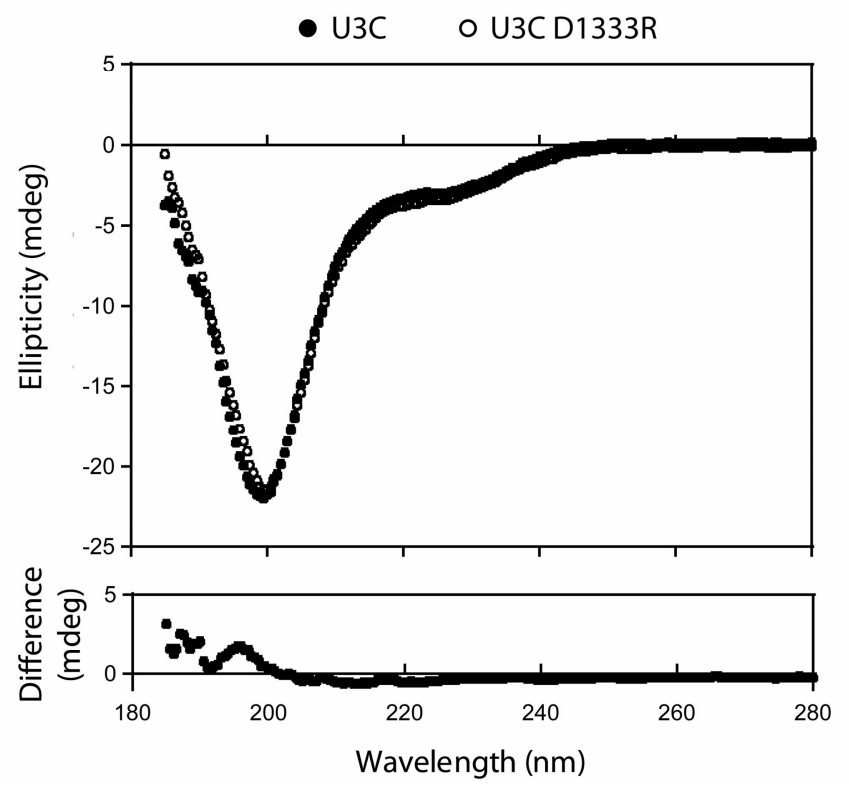


Figure 5. The point mutation D1333R in the U3C fragment inhibits its WASH displacement ability.

(A) 3T3 cells were transiently transfected with PC-mCherry fused to wild type or mutant U3C. After 2 days, cells were processed for immunofluorescence using WASH antibodies, and observed by epifluorescence microscopy. Endogenous WASH appears displaced by the

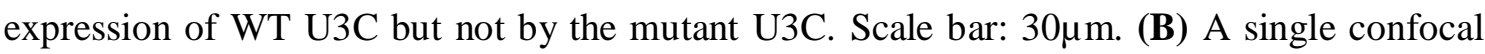
section is displayed. Endogenous WASH is indeed displaced from transferrin-positive endosomes by the expression of WT U3C but not by the mutant U3C. Scale bar: $10 \mu \mathrm{m}(1 \mu \mathrm{m}$ in inserts). (C) Percentage of cells displaying clear displacement of endosomal WASH (black), no displacement (white) or an ambiguous displacement (grey) (n: total number of transfected cells observed for each construct). The percentage values (mean $+/-$ sem) were averaged over 2 independent experiments. (D) Expression levels of WT and mutant U3C or full length FAM21 in transiently transfected $3 \mathrm{~T} 3$ cells. The arrows indicate the positions of FAM21 U3C and FL. The D1333R mutation had inverse effect in U3C and FL FAM21. In the U3C context, many more cells express the mutant, whereas in the FL context, the mutation impairs FAM21 expression.

A

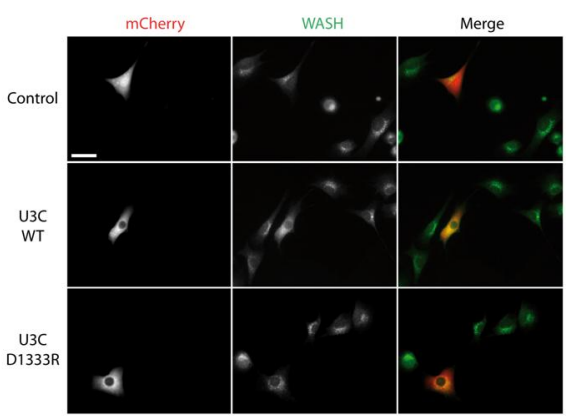

B

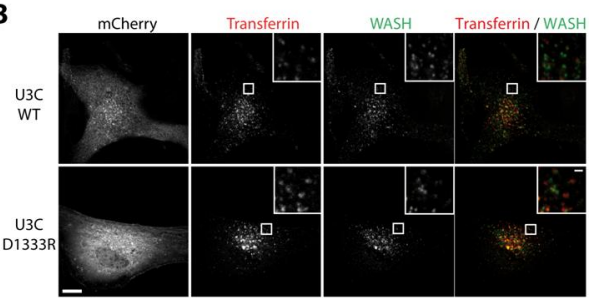

C

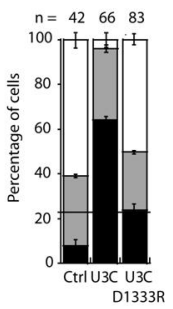

D

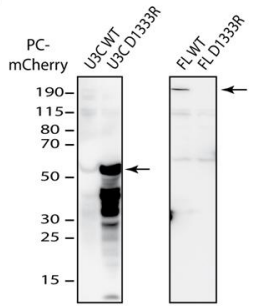


Figure 6. FAM21 active fragments impair colocalization of WASH with the Cargo Selective Complex

(A) $3 \mathrm{~T} 3$ cells were transiently transfected with the indicated PC-mCherry tagged constructs. After 1 day, transfected cells were processed for immunofluorescence using WASH and VPS35 antibodies and observed by confocal microscopy. A single confocal section is displayed. In control cells, many WASH punctae colocalize with VPS35 punctae. In contrast, in cells that overexpress FAM21 FL or U3C, WASH staining is more diffuse and most WASH punctae do not colocalize with VPS35 punctae. The D1333R mutation in U3C partially restores the association between WASH and VPS35. Scale bar: $10 \mu \mathrm{m}(1 \mu \mathrm{m}$ in inserts). (B) Colocalization is given as the percent of WASH objects that have an associated VPS35 object (within $290 \mathrm{~nm}$, see material and methods for details). The values (mean +/sem) were obtained from 7 to 10 cells per condition (total number of detected WASH spots: 1313 for control, 4419 for FAM21 FL, 5763 for U3C, 1206 for U3C D1333R; *: p < 0.01, **: $\mathrm{p}<0.001$, ANOVA test).

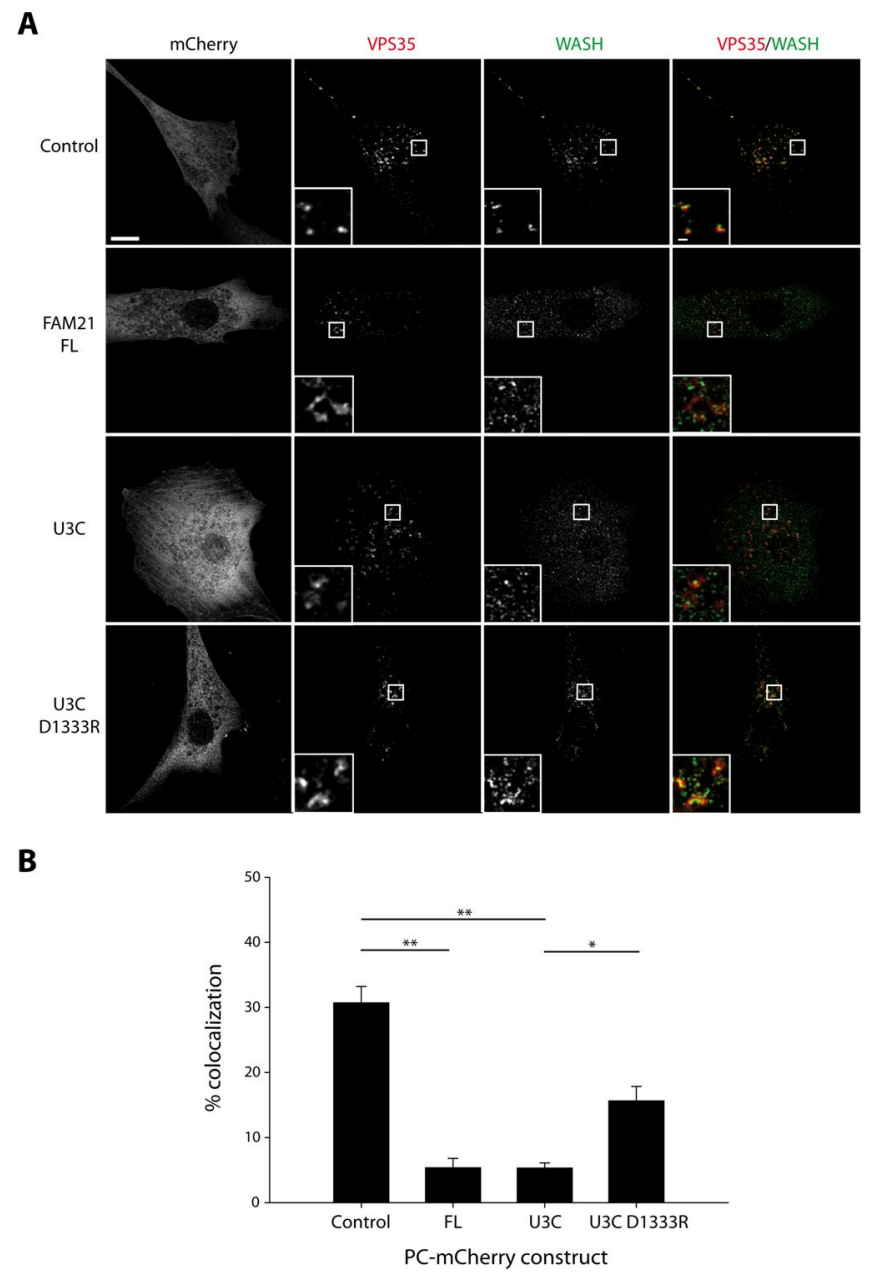


Figure 7. Additional sequences upstream of the U3C mediate binding of FAM21 to the Cargo Selective Complex.

HeLa cells were transiently transfected with the FAM21 constructs fused to mCherry. After 2 days, cells were lysed and the mCherry fusion proteins were precipitated. Precipitates were resolved by SDS-PAGE and either stained with coomassie blue or immunoblotted with the indicated antibodies. The asterisks in the coomassie stained gel indicate the positions of the different mCherry fusion proteins. The Cargo Selective Complex coprecipitates with the FAM21 fragments that displace efficiently WASH from the endosomes. However, U3C and fragments containing it are not the only fragments interacting with the CSC. For example, U1 and U2 also interact with the CSC. The D1333R mutation impairs the ability of U3C to associate with CSC, but does not abolish it. The D1333R mutation decreases the expression of full length FAM21. In order to compare, 3 fold more transfected cells were analysed with the mutant than with the wild type FAM21.

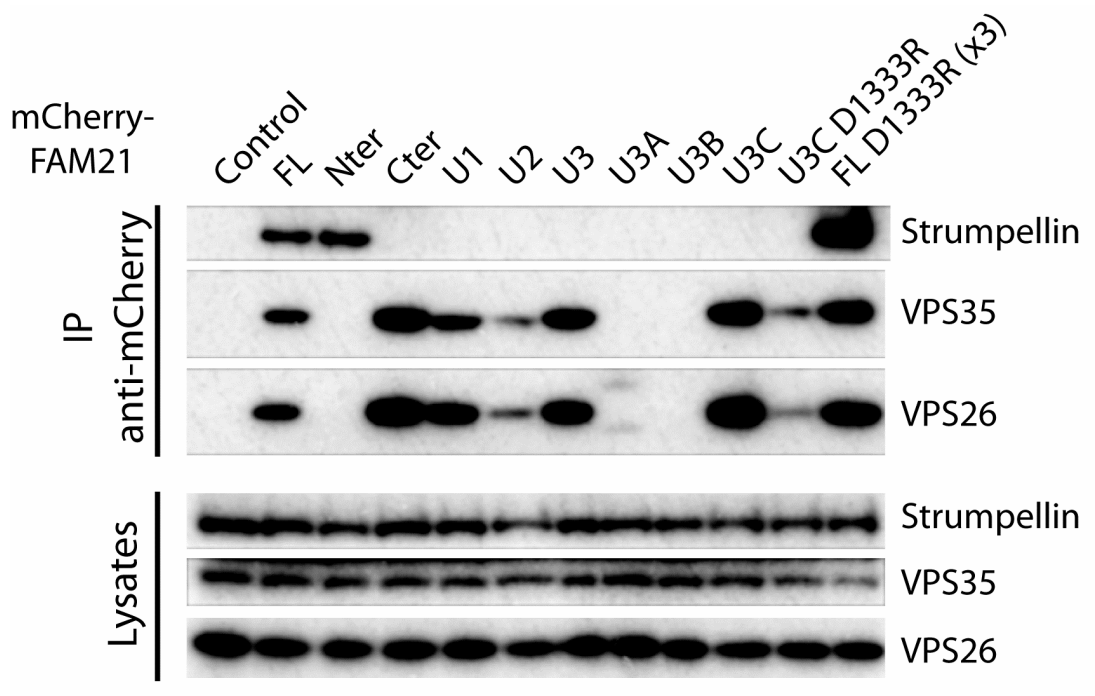

lane $\begin{array}{llllllllllllll}1 & 2 & 3 & 4 & 5 & 6 & 7 & 8 & 9 & 10 & 11 & 12\end{array}$

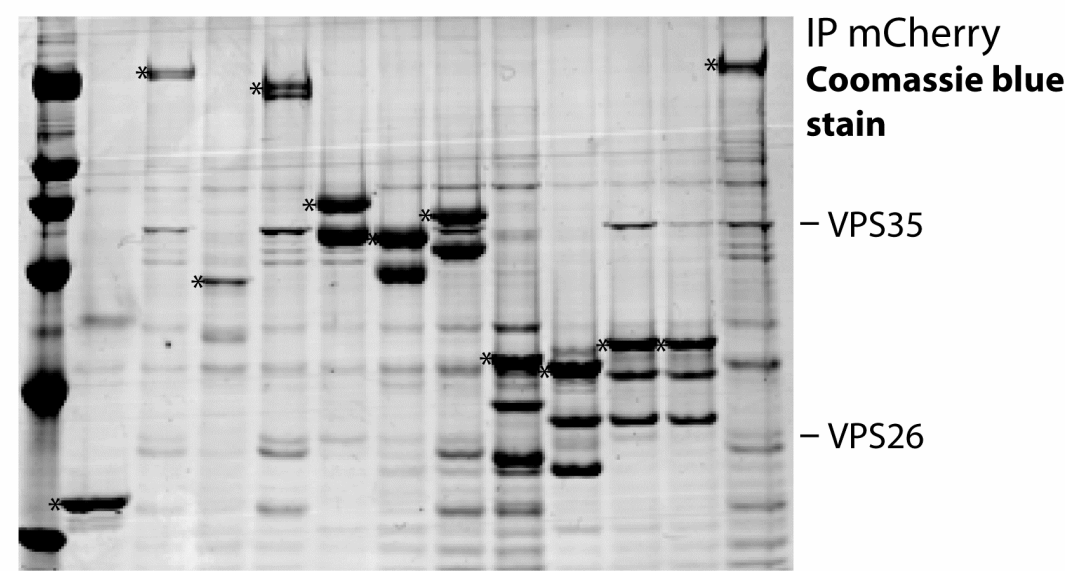




\section{Figure 8. Structural context of VPS35 His675 and VPS29 Ile91.}

(A) The structure of the VPS29 complex with the C-terminal part of the VPS35 ((Hierro et al., 2007) pdb : 2R17 ) was depicted in a cartoon representation. Residues His675 of VPS35 and Ile91 of VPS29 are represented as pink or cyan spheres, respectively. These residues face each other at the VPS35-VPS29 interface. (B) Blow-up view of the contact with representations of the side chains of the most significant residues. His675 of VPS35 forms hydrophobic contacts with both Ile91 and Pro92 of VPS29 and a weak hydrogen bond with the carbonyl function of Ile91. Val90 does not face the interface, but is represented because it is also mutated in this study. This figure was obtained with the PyMOL software.

A

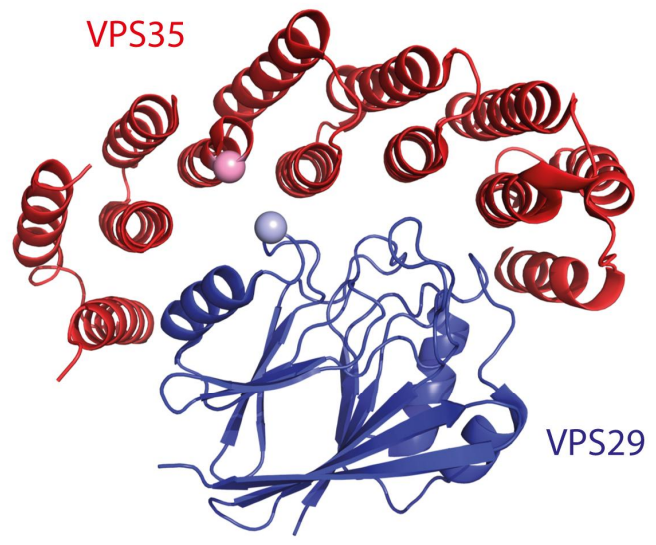

B

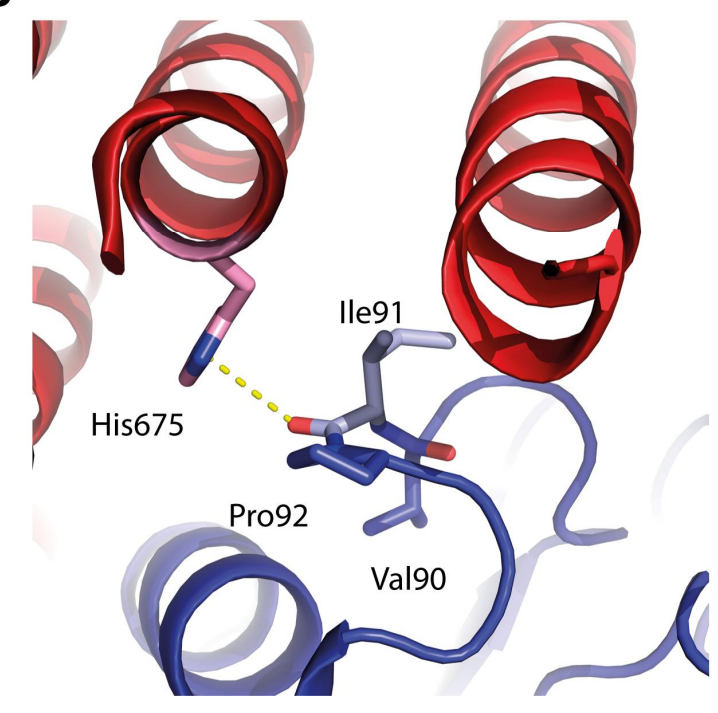




\section{Figure 9. A point mutation in VPS35 abolishes binding to FAM21.}

HeLa cells were transiently transfected with the indicated GFP fusion proteins. 2 days after transfection, cells were lysed and the lysates were immunoprecipitated with anti-GFP antibodies. Immunoprecipitates were resolved by SDS-PAGE and either silver stained or immunoblotted with the indicated antibodies. The asterisks in the silver stained gel indicate the positions of the GFP target proteins. The L108P mutation prevents VPS35 from binding to VPS26, but had no effect on the interaction with the WASH complex containing the FAM21, Strumpellin, SWIP and WASH subunits. Conversely, the H675R mutation strongly impairs association with VPS29 and the WASH complex. In this experiment, both the VPS29-GFP and FAM21-GFP constructs co-immunoprecipitated with WASH complex subunits and served as positive controls.

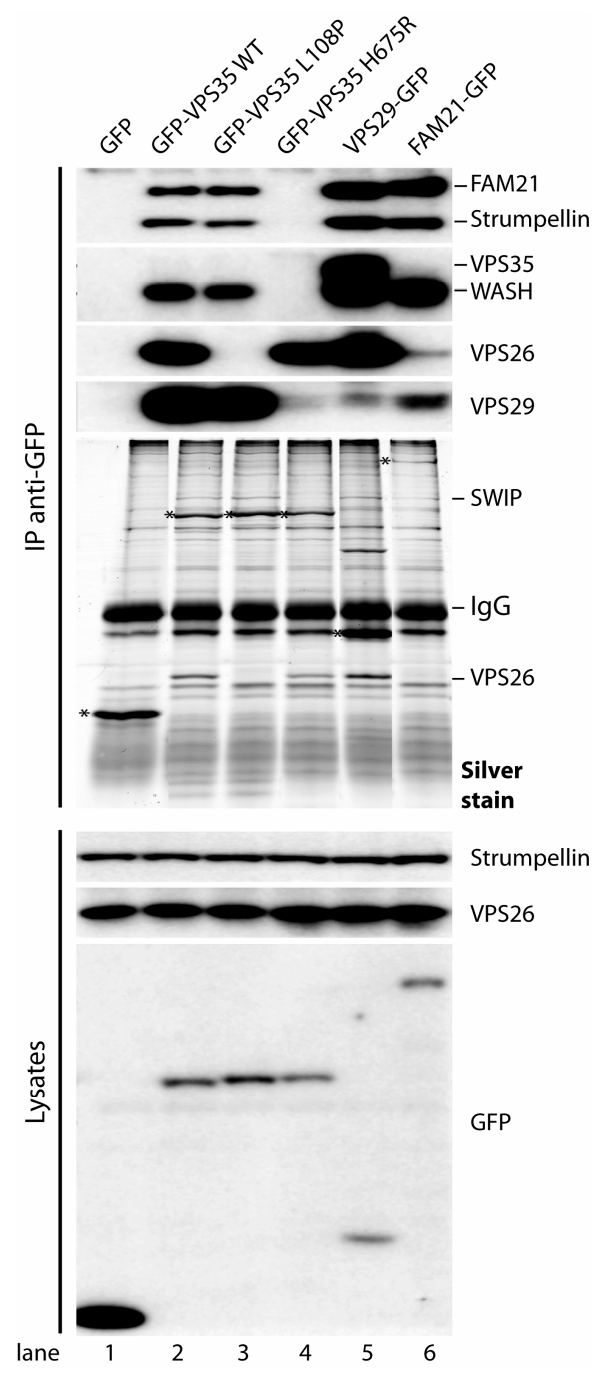

Figure 10. VPS29 binding to VPS35 is required for VPS35 interaction with FAM21. 
HeLa cells stably transfected with wildtype, V90D, I91S, L152E VPS29-GFP were transiently transfected with mCherry-VPS35 wildtype, H675R(HR), or with the empty plasmid (0), as indicated. 2 days after transfection, cells were lysed and the lysates were immunoprecipitated with anti-GFP antibodies. Immunoprecipitates were resolved by SDSPAGE and either silver stained or immunoblotted with the indicated antibodies. The asterisks in the silver stained gel indicate the position of mCherry-VPS35. The increased expression of wildtype VPS35 in the cell line expressing the VPS29 V90D mutant can overcome the weakened association between VPS29 V90D and VPS35 and restore WASH complex recruitment. But both VPS35 H675R and VPS29 I91S are inactive in this assay.

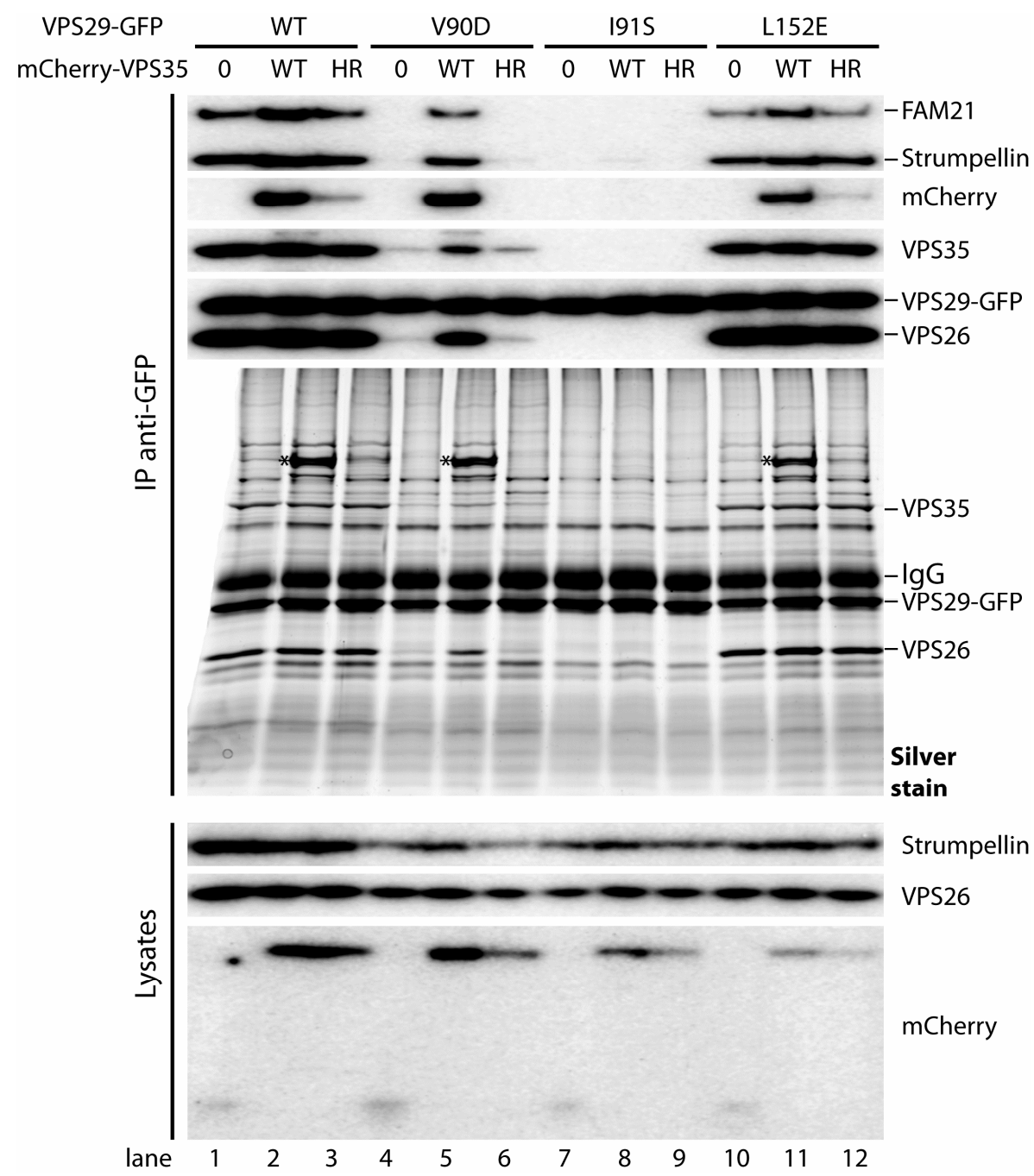

